\title{
Kinase activity of the $d g k$ gene product is involved in the virulence of Streptococcus mutans
}

\section{Correspondence \\ Yoshihisa Yamashita \\ yoshi@dent.kyushu-u.ac.jp}

Received 9 September 2008

Revised 31 October 2008

Accepted 2 November 2008
Yukie Shibata, ${ }^{1}$ Jan R. van der Ploeg, ${ }^{2}$ Takeshi Kozuki, ${ }^{3}$ Yasuhito Shirai, ${ }^{3}$ Naoaki Saito, ${ }^{3}$ Miki Kawada-Matsuo, ${ }^{1}$ Toru Takeshita ${ }^{1}$ and Yoshihisa Yamashita ${ }^{1}$

\author{
${ }^{1}$ Department of Preventive Dentistry, Kyushu University Faculty of Dental Science, 3-1-1 Maidashi, \\ Higashi-ku, Fukuoka 812-8582, Japan \\ ${ }^{2}$ Institute for Oral Biology, University of Zürich, Plattenstrasse 11, CH-8032 Zürich, Switzerland \\ ${ }^{3}$ The Biosignal Research Center, Kobe University, 1-1 Rokkodai-cho, Nada-ku, Kobe 657-8501, \\ Japan
}

C-terminal deletion of the diacylglycerol kinase (Dgk) homologue of the cariogenic oral bacterium Streptococcus mutans resulted in loss of aciduricity. To confirm the role of the $\mathrm{C}$ terminus of the Dgk homologue in aciduricity, various mutants of S. mutans UA159 with a C-terminally truncated Dgk homologue were constructed. The deletion of one or two amino acid residues at the $\mathrm{C}$ terminus had no effect on the acid-tolerance properties of mutants. When further amino acid residues at the $\mathrm{C}$ terminus were removed, mutants became more acid-sensitive. The mutant with deletion of eight amino acid residues at the $\mathrm{C}$ terminus did not grow at $\mathrm{pH} 5.5$, suggesting that the C-terminal tail of the Dgk homologue was indispensable for tolerance to acid stress in $S$. mutans. Kinase activity assays revealed that deletion of the C-terminal amino acids of Dgk led to a reduction of kinase activity for undecaprenol. A truncated mutant that had completely lost kinase activity was unable to grow at $\mathrm{pH}$ 5.5. These results suggest that the acid tolerance of $S$. mutans is closely related to kinase activity of the Dgk homologue. Additionally, the $d g k$ deletion mutant exhibited markedly reduced levels of smooth-surface carious lesions in pathogen-free rats, despite there being no difference between the mutant and the parental organism in the extent of total smooth surface plaque. The results suggest that Dgk activity may play a direct role in the virulence of $S$. mutans.

\section{INTRODUCTION}

Streptococcus mutans is the major aetiological agent of human dental caries (Loesche, 1986) and is capable of forming a biofilm known as dental plaque on the tooth surface (Tanzer et al., 2001). Within dental plaque, $S$. mutans can produce large amounts of acids from fermentable dietary carbohydrates. Acid accumulation can eventually dissolve the hard, crystalline structure of the teeth, resulting in a carious lesion (Quivey et al., 2001). This bacterium has the ability to tolerate the low $\mathrm{pH}$ in dental plaque. This property is considered to be one of the most important virulence factors of this micro-organism. Our previous work demonstrated that the diacylglycerol kinase (Dgk) homologue of S. mutans is important for the response of the organism to acid stress (Yamashita et al., 1993).

Dgk catalyses the ATP-dependent phosphorylation of $s n$ 1,2-diacylglycerols and generates phosphatidic acid. In eukaryotic cells, diacylglycerol and phosphatidic acid act as

Abbreviation: Dgk, diacylglycerol kinase. second messengers in the immediate cellular response to extracellular signals (Moolenaar et al., 1986; Murayama \& Ui, 1987; Nishizuka, 1984; Topham \& Prescott, 1999). Therefore, Dgk is thought to be one of the key enzymes involved in cellular signal transduction. In bacteria, only Dgk from Escherichia coli has been well characterized. E. coli Dgk is a small integral membrane protein with a molecular mass of only $13.2 \mathrm{kDa}$; it functions in the recycling of diacylglycerol produced during the turnover of membrane phospholipids (Hasin \& Kennedy, 1982; Rotering \& Raetz, 1983). Moreover, E. coli Dgk plays an important physiological role under conditions of environmental stress, similar to eukaryotic cells (Raetz \& Newman, 1979; Walsh et al., 1986).

The S. mutans Dgk homologue is a protein of 137 amino acids with a molecular mass of $15.3 \mathrm{kDa}$. Insertion of transposon Tn916 within the codon of the tenth amino acid from the $\mathrm{C}$ terminus of the Dgk homologue causes defective growth of the resulting mutant GS5Tn1 at low $\mathrm{pH}$ (Yamashita et al., 1993). In addition, this mutant is sensitive 
to high osmolarity and elevated temperature (Yamashita et al., 1993). Chen et al. (1998) reported that the S. mutans mutant T8-1 with a similar deletion of the Dgk homologue was defective in lantibiotic mutacin II production, which is related to an environmental stress response. The $\mathrm{C}$ terminus of the Dgk homologue might be involved in signal transduction. Lis \& Kuramitsu (2003) reported that Cterminal deletion of the Dgk homologue resulted in loss of kinase activity in S. mutans cell extracts and speculated that this deletion leads to significantly lower expression of the $d g k$ gene. However, the function of the $\mathrm{C}$ terminus on kinase activity in S. mutans cell extracts is still unclear. It is of interest to study in detail the contribution of the $\mathrm{C}$ terminus of the Dgk homologue in aciduricity of S. mutans. In this study, we constructed various mutants with a C-terminally truncated Dgk homologue in S. mutans UA159 and characterized the acid tolerance of the mutants and the kinase activity of the truncated Dgk homologues. In addition, the role of the Dgk homologue in caries induction was examined.

\section{METHODS}

Bacterial strains and culture conditions. The bacterial strains and plasmids used in this study are listed in Table 1. E. coli strain RZ6 is the $d g k$ mutant of parental K12 strain R4440 (Raetz \& Newman, 1978). These strains were maintained and grown routinely as described previously (Shibata et al., 2002). Antibiotics were used at the following concentrations: $200 \mu \mathrm{g}$ erythromycin $\mathrm{ml}^{-1}, 50 \mu \mathrm{g}$ ampicillin $\mathrm{ml}^{-1}$ for E. coli; $10 \mu \mathrm{g}$ erythromycin $\mathrm{ml}^{-1}$ for S. mutans.

DNA manipulation. Standard DNA recombinant procedures, such as DNA isolation, endonuclease restriction, ligation and agarose gel electrophoresis were carried out as described by Sambrook \& Russell (2001). Transformation of S. mutans and E. coli was carried out as described previously (Hanahan, 1983; Perry et al., 1983).

Construction of $\mathbf{S}$. mutans dgk deletion mutants. We constructed various deletion mutants of the $d g k$ gene in S. mutans UA159 using double-crossover events. The primers used to construct and confirm the gene deletion are listed in Table 2 and Fig. 1. In order to construct the deletions followed immediately by termination codons, the reverse primers contained the termination codon (TAA) in an appropriate site. Fig. 1(b) shows the strategy for construction of UADGK1 as an example. First, the $\mathrm{Em}^{\mathrm{r}}$ fragment containing an erythromycin-resistance gene from plasmid pResEmNot (Shiroza \& Kuramitsu, 1993) was amplified using Em-F and Em-R primers with HindIII and PvuII sites, respectively, engineered into their $5^{\prime}$ ends. The $\mathrm{Em}^{\mathrm{r}}$ fragment was cloned into HindIII- and EcoRV-digested pBluescript SK II $(+)$, yielding pBSSKII-Em ${ }^{\mathrm{r}}$. Next, the Sgp fragment containing a $3^{\prime}$ BamHI site, which consisted of the downstream sequence of the $d g k$ gene, including the $\mathrm{SD}$ sequence and full sequence of the $\operatorname{sg} p$ gene, was amplified using SGP-F and SGP-R primers. This

Table 1. Bacterial strains and plasmids used in this study

\begin{tabular}{|c|c|c|}
\hline Strain or plasmid & Relevant characteristics & Source or reference \\
\hline \multicolumn{3}{|l|}{ E. coli } \\
\hline DH5 $\alpha$ & supE44 $\Delta$ lacU169 ( $\phi 80$ lacZAM15) hsdR17 recA1 endA1 gyrA96 thi-1 relA1 & Sambrook \& Russell (2001) \\
\hline RZ6 & $d g k-6$ derivative (defective diglyceride kinase) of R4440 & Raetz \& Newman (1978) \\
\hline RZQE80 & RZ6 carrying pQE80L & This study \\
\hline RZDGK1 & RZ6 carrying pQEDgk1 & This study \\
\hline RZDGK4 & RZ6 carrying pQEDgk4 & This study \\
\hline RZDGK6 & RZ6 carrying pQEDgk6 & This study \\
\hline RZDGK11 & RZ6 carrying pQEDgk11 & This study \\
\hline \multicolumn{3}{|l|}{ S. mutans } \\
\hline UA159 & Serotype c wild-type strain & \\
\hline UADGK1 & Em $^{\mathrm{r}}$; UA159 expressing Dgk1 & This study \\
\hline UADGK2 & Em $^{\mathrm{r}}$; UA159 expressing Dgk2 & This study \\
\hline UADGK3 & Em $^{\mathrm{r}}$; UA159 expressing Dgk3 & This study \\
\hline UADGK4 & $\mathrm{Em}^{\mathrm{r}}$; UA159 expressing Dgk4 & This study \\
\hline UADGK5 & $\mathrm{Em}^{\mathrm{r}}$; UA159 expressing Dgk5 & This study \\
\hline UADGK6 & Em $^{\mathrm{r}}$; UA159 expressing Dgk6 & This study \\
\hline UADGK7 & $\mathrm{Em}^{\mathrm{r}}$; UA159 expressing Dgk7 & This study \\
\hline UADGK8 & Em $^{\mathrm{r}}$; UA159 expressing Dgk8 & This study \\
\hline UADGK9 & Em $^{\mathrm{r}}$; UA159 expressing Dgk9 & This study \\
\hline UADGK10 & $\mathrm{Em}^{\mathrm{r}}$; UA159 expressing Dgk10 & This study \\
\hline UADGK11 & Em $^{\mathrm{r}}$; UA159 expressing Dgk11 (full-size Dgk) & This study \\
\hline UADGK12 & Em $^{\mathrm{r}}$; UA159 expressing Dgk12 & This study \\
\hline UADGK13 & $\mathrm{Em}^{\mathrm{r}}$; UA159 expressing Dgk13 & This study \\
\hline \multicolumn{3}{|l|}{ Plasmids } \\
\hline pQE80L & $A p^{r}$; expression vector & Qiagen \\
\hline pQEDgk1 & pQE80L expressing Dgk1 & This study \\
\hline pQEDgk4 & pQE80L expressing Dgk4 & This study \\
\hline pQEDgk6 & pQE80L expressing Dgk6 & This study \\
\hline pQEDgk11 & pQE80L expressing Dgk11 (the full-size Dgk) & This study \\
\hline
\end{tabular}


Table 2. Primers used in this study

Nucleotides underlined in each primer sequence show the positions of the restriction endonuclease sites incorporated to facilitate cloning, and termination codons are in bold.

\begin{tabular}{|c|c|c|}
\hline Primer & Sequence $\left(5^{\prime}-3^{\prime}\right)$ & Reference \\
\hline SGP-F & AAATAGATAGAGGAGACATC & This study \\
\hline SGP-R & CGCGGATCCACCGACTTTTGAGCGGAT & This study \\
\hline DGK-F1 & CGCCTCGAGTGAAATGATTGATGAGAC & This study \\
\hline DGK-F2 & CGCGGATCCCCTATGGACTTAAGAGAT & This study \\
\hline DGK-R1 & CGCAAGCTTTTAAAAAATAATCAAGCCTGTCA & This study \\
\hline DGK-R2 & CGCAAGCTTTTATACAAAAATAATCAAGCCTG & This study \\
\hline DGK-R3 & CGCAAGCTTTTATGGTACAAAAATAATCAAGC & This study \\
\hline DGK-R4 & CGCAAGCTTTTATTTTGGTACAAAAATAATCAZ & This study \\
\hline DGK-R5 & CGCAAGCTTTTAAATTTTTGGTACAAAAATAA & This study \\
\hline DGK-R6 & CGCAAGCTTTTACCAAATTTTTGGTACAAAAA & This study \\
\hline DGK-R7 & CGCAAGCTTTTAAAACCAAATTTTTGGTACAA & This study \\
\hline DGK-R8 & CGCAAGCTTTTACAAAAACCAAATTTTTGGTA & This study \\
\hline DGK-R9 & CGCAAGCTTTTAAAGCAAAAACCAAATTTTTG & This study \\
\hline DGK-R10 & CGCAAGCTTTTAAAAAAGCAAAAACCAAATTT & This study \\
\hline DGK-R11 & CGCAAGCTTTTAATGAAAAAGCAAAAACCAAA & This study \\
\hline DGK-R12 & CGCAAGCTTTTAATGATAGTCACTGGCCAAAT & This study \\
\hline DGK-R13 & CGCAAGCTTTTAATTATCTCTTAAGTCCATAG & This study \\
\hline Em-F & CGCAAGCTTGAAGCAAACTTAAGAGTG & This study \\
\hline Em-R & CGCCAGCTGTTATTTCCTCCCGTTAAA & This study \\
\hline DGKRT-1 & CCAGCCTTGAATTCGCTTTAA & This study \\
\hline DGKRT-2 & ACCAAACCAGCAATGACAGCTA & This study \\
\hline 16SRT-1 & CTTACCAGGTCTTGACATCCCG & Korithoski et al. (2007) \\
\hline 16SRT-2 & ACCCAACATCTCACGACACGAG & Korithoski et al. (2007) \\
\hline
\end{tabular}

(a)

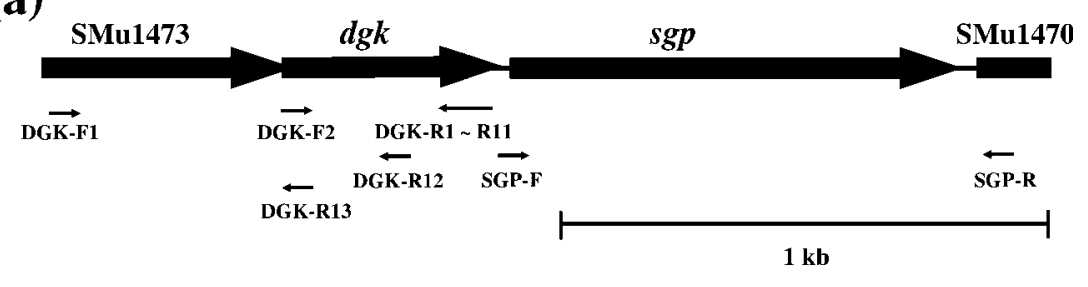

(b)

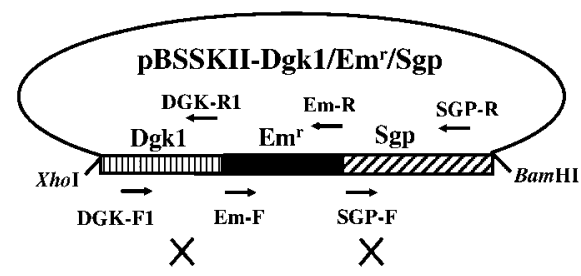

UA159

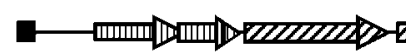

SMu1473 dgk sgp SMu1470

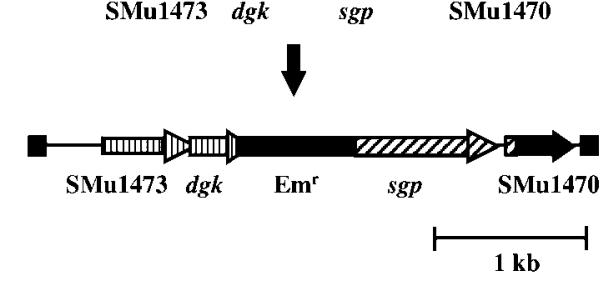

UADGK1

Fig. 1. (a) Diagram of the dgk locus from $S$. mutans UA159. Relevant primers for construction of the $d g k$ deletion mutants are shown by small arrows. (b) Construction of the $d g k$ deletion mutant UADGK1 by a double-crossover event. The mutagenesis procedure is described in Methods. 
fragment was inserted into SmaI/BamHI-digested pBSSKII-Em ${ }^{\mathrm{r}}$, to yield pBSSKII-Em ${ }^{\mathrm{r}} / \mathrm{Sgp}$. Finally, the Dgk1 fragment was amplified with the primer pair DGK-F1/DGK-R1 (Fig. 1b). The amplified fragment was digested with XhoI and HindIII and then cloned into

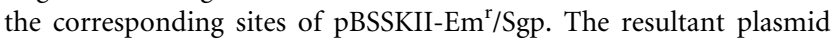
was designated pBSSKII-Dgk1/Em ${ }^{\mathrm{r}} / \mathrm{Sgp}$. The other plasmids, pBSSKII-Dgk2-13/Em ${ }^{\mathrm{r}} / \mathrm{Sgp}$, were constructed using the same strategy with the primer DGK-R2-13 as the reverse primer. These plasmids were individually digested with BamHI and XhoI, and assembled fragments were then transformed into $S$. mutans UA159. The mutants obtained were designated UADGK1-13 (Table 1), expressing Dgk113, respectively, as shown in Fig. 2. Correct replacements of transformants were confirmed by sequencing or PCR.

Evaluation for acid sensitivity of strains. Overnight cultures of the strains were diluted into brain heart infusion (BHI) broth and incubated at $37{ }^{\circ} \mathrm{C}$ in $5.0 \% \mathrm{CO}_{2}$ until mid-exponential-phase $\left(\mathrm{OD}_{550}\right.$ $\sim 0.4)$. Samples $(20 \mu \mathrm{l})$ of mid-exponential phase cells for the strains were inoculated in microtitre plate wells containing $200 \mu \mathrm{BHI}$ broth at $\mathrm{pH} 7.45$ and $\mathrm{pH}$ 5.5. Growth was monitored at $550 \mathrm{~nm}$ using a Spectramax 340 $\mathrm{PC}^{384}$ microplate spectrophotometer (Molecular Devices). From the data obtained, growth curves were generated and the mid-exponential-phase doubling time $\left(T_{\mathrm{d}}\right)$ was determined.

Construction of $E$. coli RZ6 strains expressing S. mutans dgk genes. The construction of plasmids (pQEDgk11, pQEDgk6, pQEDgk4 and pQEDgk1; Table 1) for expression of S. mutans $d g k$ in E. coli was carried out as described below. A DNA fragment containing the $S$. mutans $d g k$ gene (wild-type form) was amplified by PCR using primer DGK-F2, starting from the second codon, and primer DGK-R11 (Table 2). The generated fragment was digested with BamHI and HindIII and inserted into the corresponding sites of vector $\mathrm{pQE} 80 \mathrm{~L}$, resulting in plasmid $\mathrm{pQEDgk11}$. A series of plasmids was constructed using a similar method except for the primer sets used: DGK-F2/DGK-R6, DGK-F2/DGK-R4 and DGK-F2/DGK-R1 for pQEDgk6, pQEDgk4 and pQEDgk1, respectively. Each plasmid was transformed into E. coli dgk mutant RZ6 (Table 1).

Immunoblotting and kinase assay. The cells were harvested, resuspended in homogenizing buffer $[50 \mathrm{mM}$ Tris/ $\mathrm{HCl}(\mathrm{pH} 7.4)$, $250 \mathrm{mM}$ sucrose, $10 \mathrm{mM}$ EGTA, $2 \mathrm{mM}$ EDTA, $200 \mathrm{mg}$ leupeptin $\mathrm{ml}^{-1}, 1 \mathrm{mM}$ PMSF, $1 \%$ Triton X-100], and sonicated twice for $30 \mathrm{~s}$ each at output setting 5, using Handy Sonic (Tomy).

For immunoblotting, the samples were subjected to $15 \%$ SDS-PAGE, followed by blotting onto a PVDF membrane (Millipore). Non- specific binding sites were blocked by incubation with $5 \%$ skim milk in $10 \mathrm{mM}$ PBS containing $0.03 \%$ Triton X-100 (PBS-T) at $4{ }^{\circ} \mathrm{C}$ overnight. The membrane was incubated with anti-His-tag antibody for $1 \mathrm{~h}$ at room temperature. After washing with PBS-T, the membrane was incubated with peroxidase-labelled anti-mouse IgG (Jackson ImmunoResearch Laboratories) for $30 \mathrm{~min}$. After three rinses with PBS-T, the immunoreactive bands were visualized using a chemiluminescence detection kit (ECL, GE Healthcare).

The kinase activity in cell lysates was examined with the octyl glucoside mixed-micelle assay (Preiss et al., 1986), using undecaprenol (Larodan Fine Chemicals) as a substrate. The reaction was performed for $10 \mathrm{~min}$ in the presence of $\left[\gamma-{ }^{32} \mathrm{P}\right] \mathrm{ATP}$. The radioactivity of undecaprenyl phosphate was separated on $20 \mathrm{~cm}$ silica gel 60 TLC plates (Merck) using chloroform/methanol/acetic acid ( $65: 15: 5$, by vol.), detected by BAS2500 (Fujix) and finally represented as photostimulated luminescence, which is the established method in this imaging system. The amount of each sample subjected to the kinase assay was determined by immunoblotting with an anti-His-tag antibody.

qRT-PCR analysis of $\boldsymbol{d g} \boldsymbol{k}$ expression. Total RNA was prepared from S. mutans strains as described previously (Shibata et al., 1999). Before the RNA solution was used for the reverse transcriptase PCR, contaminating DNA was eliminated by digestion with RNase-free DNase (Nippon Gene). cDNA was generated via reverse transcription using a high-capacity cDNA reverse transcription kit (Applied Biosystems) according to the supplier's instructions. RNA samples lacking reverse transcriptase were included as controls to ensure that results were not the product of residual DNA contamination. Singlestranded cDNA template quantitative real-time PCRs (qRT-PCRs) were performed using the QuantiTect SYBR green PCR kit (Qiagen) in a StepOne real-time PCR system (Applied Biosystems). The primer sequences used in the analysis are listed in Table 2. Results were normalized against the expression of an internal standard, 16S rRNA. Relative expression levels were calculated using the $2^{-\Delta \Delta \mathrm{CT}}$ method (Livak \& Schmittgen, 2001).

Cariogenic potential, plaque formation and establishment of $\boldsymbol{S}$. mutans wild-type and mutant strains. The animal study was approved by the 'Veterinäramt des Kantons Zürich' and conformed to the Swiss laws on animal protection. Specific-pathogen-free, cariessusceptible Osborne-Mendel rats (Institute for Oral Biology, University of Zürich, Switzerland) were used to investigate in vivo effects of the $d g k$ deletion on smooth-surface dental plaque, smoothsurface caries, and initial and advanced dentinal fissure caries, as well as on the establishment of $S$. mutans in the oral microbiota. Each

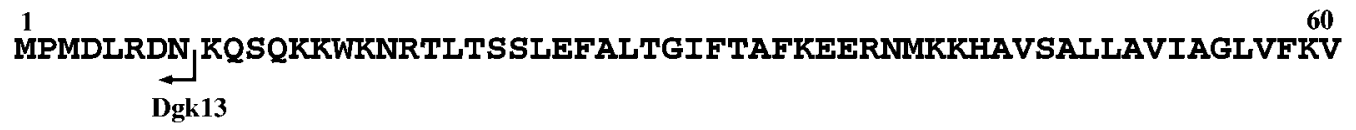

61

SVIEWLFLLLSIFLVITFEIVNSAIENVVDLASDYH FSMLAKNAKDMAAGAVLVISGFAA

Dgk12

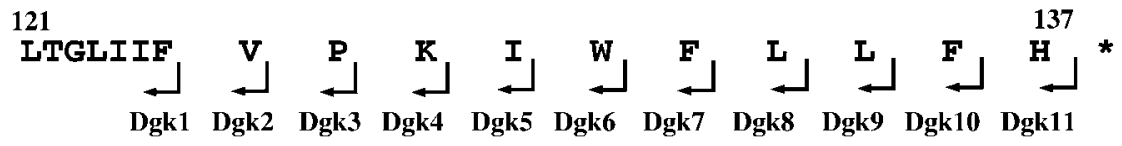

Fig. 2. Representation of the truncated Dgk proteins used. The deduced amino acid sequence of the dgk gene from $S$. mutans UA159 is presented. The terminal amino acid of the truncated Dgk expressed in each UA159 dgk mutant and each E. coli RZ transformant is indicated along the sequences by a right-angled arrow, which indicates that the sequence is deleted from the right up to this site. 
experimental group consisted of ten animals. Thirteen days after birth, the pups and their dams were transferred to stainless-steel screen-bottom cages without bedding and fed a finely ground stock diet (diet no. 3433 Provimi Kliba AG, Switzerland) to prevent impaction of food and bedding particles in fissures. Tap water and food were given ad libitum. On day 20 after birth, the dams were removed and the littermates distributed among three treatments, with two animals per cage. The cages and feeding equipment had been sterilized prior to being occupied by the animals. The animals were placed in a separate rack as far away as possible from the other racks in order to minimize the risk of cross-infection.

On days 21 and 22, each rat was infected orally, twice daily, by inoculation of $200 \mu \mathrm{l}$ heavy suspensions of the two bacterial strains (the wild-type strain UA159 and the $d g k$ mutant strain UADGK1, Table 1). The uninfected control group was given $200 \mu \mathrm{l}$ distilled water. To support the implantation of these bacteria, all rats, including the noninfected control group, received drinking water containing $2 \%$ sucrose and $2 \%$ glucose on days 20 to 22 as well as the cariogenic diet 2000a containing $40 \%$ sucrose. On day 23 , sterilization of the feeding and housing equipment was resumed. The trays containing urine and faeces were sterilized daily, drinking bottles and food cups were sterilized every other day, and all cages were sterilized weekly. Hand disinfection and fresh disposable aprons were used between handling of the different treatment groups. Shortly before the end of the study, oral swabs were taken from all 30 rats to obtain a final determination of the microbial status of the animals. Microbiological analysis of the swabs was performed as described previously (Senadheera et al., 2005).

On day 52, the animals were anaesthetized with $\mathrm{CO}_{2}$ and decapitated. The upper and lower jaws were dissected out and immersed in fixative ( $10 \%$ buffered formalin phosphate) for a minimum of $72 \mathrm{~h}$. Erythrosine-stained maxillary molars were evaluated for plaque extent using the method described by Regolati \& Hotz (1972), and mandibular molars were sectioned and scored for fissure caries (König et al., 1958). Smooth-surface carious lesions were scored as described previously (Keyes, 1958a, b).

Statistical analysis. A one-way analysis of variance (ANOVA) was used to determine the significance of differences among groups, followed by Bonferroni multiple comparison test. Differences were considered significant only for values of $P<0.05$.

\section{RESULTS}

\section{Acid sensitivity of the dgk deletion mutants}

To evaluate the contribution of the $\mathrm{C}$ terminus of Dgk to acid tolerance, we constructed an 11-mutant panel in which the Dgks were sequentially truncated by one amino acid from the $\mathrm{C}$ terminus (Table 1, Fig. 2). The mutant UADGK11, which had the $\mathrm{Em}^{\mathrm{r}}$ fragment immediately downstream of the $d g k$ gene, was constructed as a control strain instead of the wild-type UA159. UADGK11 had the same ability to tolerate low $\mathrm{pH}$ as the wild-type strain (data not shown). All mutant strains grew similarly at $\mathrm{pH} 7.45$ and had doubling times of 53.8-61.6 min, with no significant difference among them (Table 3). However, they exhibited different growth rates when grown at $\mathrm{pH} 5.5$ (Table 3, Fig. 3). UADGK8, in which the C-terminal three amino acid residues of Dgk were deleted, had a slightly reduced growth rate. Subsequent deletion of the amino acid resulted in mutants further reduced in growth rate. When seven amino acid residues were removed, UADGK4
Table 3. Effect of low $\mathrm{pH}$ on growth of S. mutans UA159 dgk mutants

Doubling time was calculated based on the formulae $\ln Z-\ln Z_{0}=k(t-$ $t_{0}$ ), where $k$ is the growth rate, and $g=0.693 / k$, where $g$ is the doubling time. Values are the mean \pm SD obtained from three independent experiments. Differences in the doubling time between UADGK11 and UADGK5-10 were analysed by Bonferroni multiple comparison test.

\begin{tabular}{|lcc|}
\hline \multirow{2}{*}{ Strain } & \multicolumn{2}{c|}{ Doubling time (min) in: } \\
\cline { 2 - 3 } & BHI pH 7.45 & BHI pH 5.5 \\
\hline UADGK11 & $57.8 \pm 4.4$ & $132.3 \pm 12.9$ \\
UADGK10 & $58.2 \pm 6.4$ & $131.2 \pm 11.7$ \\
UADGK9 & $61.2 \pm 5.8$ & $133.6 \pm 11.7$ \\
UADGK8 & $61.6 \pm 1.8$ & $177.6 \pm 19.0$ \\
UADGK7 & $55.5 \pm 3.4$ & $202.5 \pm 24.4^{*}$ \\
UADGK6 & $57.5 \pm 2.5$ & $248.9 \pm 32.2 \dagger$ \\
UADGK5 & $55.3 \pm 5.4$ & $271.3 \pm 34.6 \ddagger$ \\
UADGK4 & $55.1 \pm 6.0$ & $>1000 \S$ \\
UADGK3 & $53.8 \pm 3.7$ & $>1000 \$$ \\
UADGK2 & $58.4 \pm 6.7$ & $>1000 \S$ \\
UADGK1 & $57.0 \pm 6.1$ & $>1000 \S$ \\
\hline
\end{tabular}

${ }^{\star}$ Statistically significantly different $(P<0.05)$ from UADGK11. $\dagger$ Statistically significantly different $(P<0.001)$ from UADGK11. $\ddagger$ Statistically significantly different $(P<0.0001)$ from UADGK11. $\S$ Statistical analyses were not carried out because growth rates were too slow.

showed extremely limited growth until $9 \mathrm{~h}$, but the final resting culture density of this mutant increased to 0.16 at $\mathrm{OD}_{550}$. The other truncated mutants, UADGK3 to UADGK1, did not grow at pH 5.5. UADGK7, UADGK6 and UADGK5 had significantly increased doubling times $(P<0.05, P<0.001$ and $P<0.0001$, respectively) compared to UADGK11. These results suggest that the $\mathrm{C}$-terminal tail of the Dgk homologue is indispensable for tolerance to acid stress in S. mutans.

As stated above, the deletion of only eight C-terminal amino acid residues of the Dgk homologue resulted in loss of the ability to grow at $\mathrm{pH} 5.5$ in the resultant mutant UADGK3. To examine the acid-sensitive phenotype of mutants with further deletion of the Dgk homologue, we constructed an additional two UA159 $d g k$ mutants, UADGK12 and UADGK13 (Table 1 and Fig. 2). These two mutants, in addition to UADGK1, were grown at $\mathrm{pH} 5.5,5.8$, or 6.3. There were negligible differences among their growth rates at all acidic pHs tested (data not shown).

\section{Kinase activity of the full-size and truncated Dgks}

The actual substrate for the $S$. mutans Dgk homologue is not diacylglycerol but undecaprenol (Lis \& Kuramitsu, 2003). Therefore, all kinase assays were performed using undecaprenol as the substrate. The E. coli strains RZDGK1, 


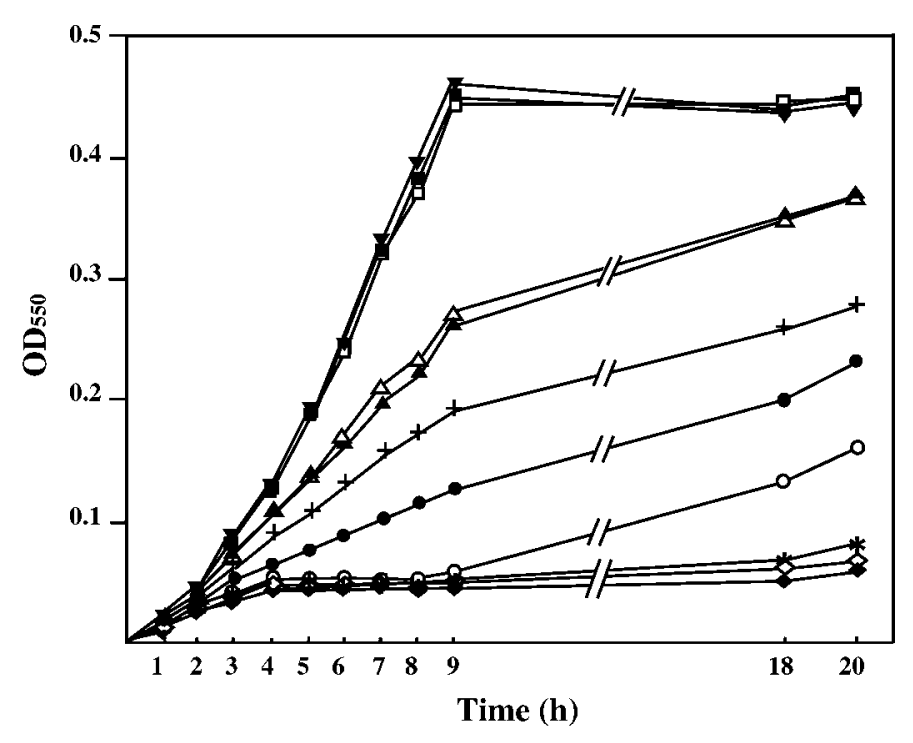

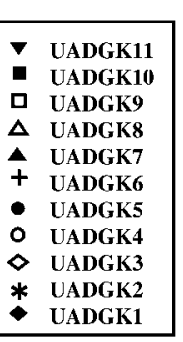

Fig. 3. Growth curves of $S$. mutans UA159 $d g k$ mutants grown in $\mathrm{BHI}$ medium at $\mathrm{pH} 5.5$. Growth was defined as the increase in $\mathrm{OD}_{550}$, calculated by subtraction of $\mathrm{OD}_{550}$ at the initiation of growth from that at the times indicated. The results are the means of three experiments.
RZDGK4, RZDGK6 and RZDGK11 produced the Dgk proteins corresponding to $S$. mutans strains UADGK1, UADGK4, UADGK6 and UADGK11, respectively (Table 1, Fig. 2). Cell lysates from E. coli strains were subjected to the octyl glucoside mixed-micelle assay using undecaprenol as the substrate (Fig. 4a). Immunoblotting using anti-His-tag antibody revealed that each mutant produced a protein of the appropriate molecular size (Fig. 4a) and that no significant degradation products were detected. As expected, the cell lysates from RZQE80 exhibited no kinase activity. In contrast, cell lysates from RZDGK11 expressing the full-size S. mutans Dgk protein catalysed transfer of a large amount of phosphate to undecaprenol. This activity was significantly stronger compared to when diacylglycerol was used as the substrate (data not shown). Cell lysates from RZDGK6 expressing the truncated Dgk with the deletion of five amino acid residues from the $\mathrm{C}$ terminus showed clear reduction of kinase activity. Furthermore, when seven amino acid residues were deleted from the $\mathrm{C}$ terminus (corresponding to RZDGK4), kinase activity became much weaker compared to that of RZDGK6. Truncated Dgk with the deletion of ten C-terminal amino acid residues in RZDGK1 had no kinase activity. Quantitative analysis confirmed these differences (Fig. 4b). The differences in kinase activity between RZDGK11 and RZDGK6 extracts and between RZDGK6 and RZDGK4 extracts were significant $(P<0.0001)$. These results indicate that deletion of the C-terminal amino acid residues of the Dgk homologue led to reduction of kinase activity.

\section{Expression profile of $d g k$}

To confirm whether the deletion of the $3^{\prime}$-terminal end affects gene expression of $d g k$ in UADGK1, $d g k$ mRNA levels in UADGK1 and UADGK11 were compared. The ratio of the normalized mRNA expression level of $d g k$ (UADGK1/UADGK11) was $1.0 \pm 0.18$. There was no distinct difference in the expression level of the $d g k$ gene between UADGK1 and UADGK11. This result, together with the fact that cell lysates from RZDGK1 had no kinase activity, implied that the deletion of the $C$ terminus might directly cause loss of kinase activity of the Dgk homologue in S. mutans.

\section{Cariogenic potential, plaque formation, and establishment of $S$. mutans wild-type and mutant strains}

The effect of deletion of the C-terminal end of Dgk was studied in a specific-pathogen-free rat model (Table 4). Compared to the water control, the wild-type strain and the dgk mutant strain displayed significantly less smooth surface plaque extent $(P<0.0001)$; however, there was no significant difference in plaque extent between treatments with the wild-type strain and the $d g k$ mutant strain. Nevertheless, the $d g k$ mutant strain displayed significantly less smooth surface caries compared to the wild-type strain $(P<0.005)$. In addition, the mutant strain also showed reduced initial dentinal fissure lesions and advanced dentinal fissure lesions compared to the wild-type strain, but this difference was not significant.

There was no significant difference in the total number of bacteria recovered from rats between the wild-type and the $d g k$ mutant. However, rats infected with the $d g k$ mutant strain contained a significantly lower number of total streptococci and total S. mutans compared to rats infected with the wild-type strain $(P<0.05)$.

\section{DISCUSSION}

E. coli Dgk is an integral membrane protein with three transmembrane segments, with the $\mathrm{N}$ terminus of the protein in the cytoplasm, the $\mathrm{C}$ terminus in the periplasmic 
(a)

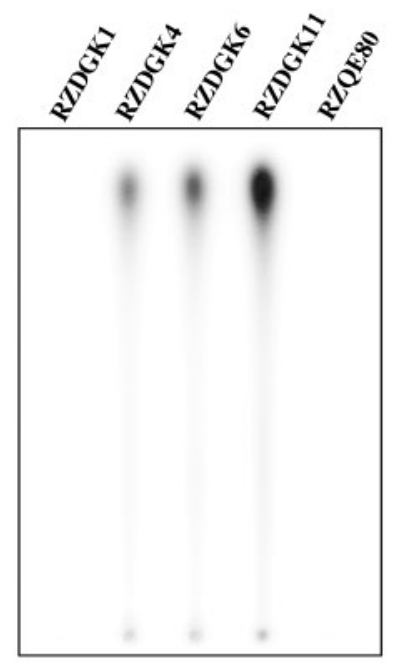

Autoradiography

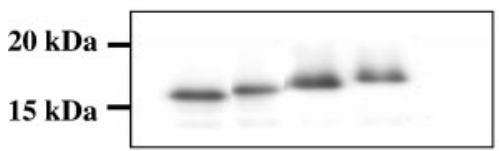

(b)

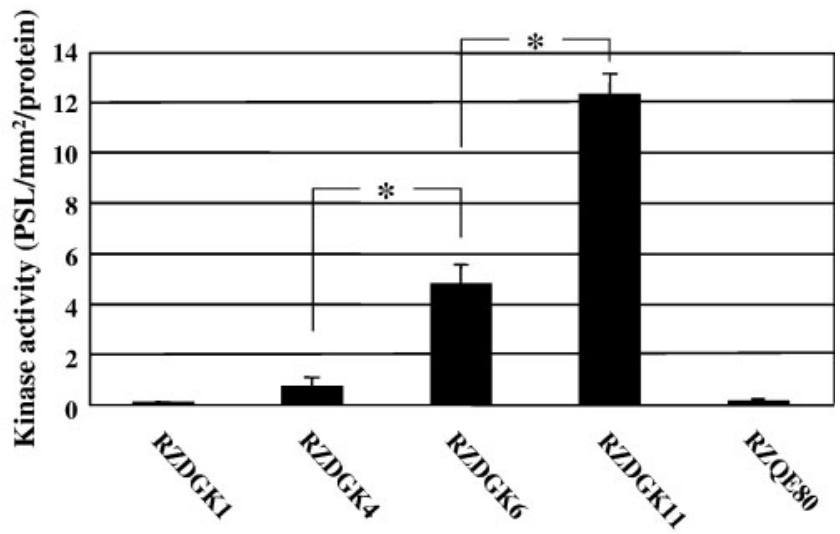

\section{Western blot: anti-His-tag}

Fig. 4. Effect of deletion of the C-terminal tail of Dgk on undecaprenol kinase activity. (a) Comparison of undecaprenol kinase activity of the full-size Dgk and various C-terminally truncated forms of Dgk. The undecaprenol kinase activity in the lysates from E. coli RZb cells was determined using an octyl glucoside mixed-micelle assay (see Methods) (top panel). Expression of the fullsize Dgk and Dgk mutants in the cell lysates is shown in the bottom panel. (b) Quantification analysis of the kinase assay. Quantification was carried out by normalization of radioactivity $\left(\mathrm{PSL} / \mathrm{mm}^{2}\right)$ in the kinase assay using the protein level detected in the Western blot. Vertical bars represent standard deviation. ${ }^{*}, P<0.0001$.

space, and two amphipathic helices near the cytoplasmic surface (Sanders et al., 1996; Smith et al., 1994). The results of multiple sequence alignment of five microbial Dgks, including S. mutans Dgk, suggest that similarities in overall sequence patterns (such as the location of hydrophobic stretches and putative amphipathic helices) that relate to the actual membrane topology might be conserved in all five Dgks (Smith et al., 1994). In addition, S. mutans Dgk has a long unique $\mathrm{C}$-terminal tail after the end of the third membrane-spanning helix (Smith et al., 1994). A previous study (Chen et al., 1998) indicated that deletion of the C terminus of the S. mutans Dgk homologue caused loss of

Table 4. Influence of $d g k$ deletion on smooth-surface plaque extent, initial and advanced dentinal fissure lesions, smooth-surface caries and colonization properties

ND, Not determined. $\Delta, 4$ units at risk; $\Delta \Delta, 12$ fissures at risk; $\Delta \Delta \Delta, 20$ units at risk.

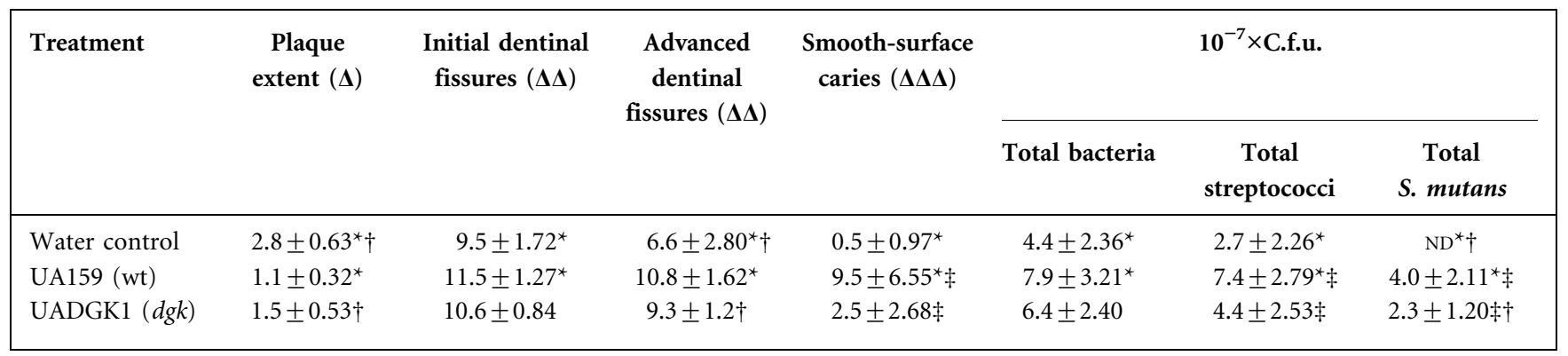

${ }^{*}$ Significant difference between water control and UA159 (wt), $P<0.05$.

$\dagger$ Significant difference between UADGK1 $(d g k)$ and water control, $P<0.05$.

$\ddagger$ Significant difference between UA159 (wt) and UADGK1 $(d g k), P<0.05$. 
production of the mutacin II lantibiotic, via decreased expression of the mutacin structural gene mutA. Considering the structure of the Dgk protein and these results, Chen et al. (1998) suggested that this unique C terminus is involved in signal transduction.

Lis \& Kuramitsu (2003) found that membranes of GS5Tn1, a $d g k$ mutant, have a very low level of kinase activity for lipid extracts from S. mutans compared to that of wild-type GS5, despite the fact that membranes of RZ6 expressing the mutated $d g k$ gene from GS5Tn1 exhibit a considerable level of activity. They supposed that disruption of the $d g k$ gene near its $3^{\prime}$ end affected expression of the gene and, consequently, caused the lack of enzymic activity in the mutant GS5Tn1. However, in our study, there was no difference in the $d g k$ mRNA level between UADGK1, expressing Dgk truncated by ten amino acids at the C terminus, and UADGK11, expressing the full-size Dgk, while the truncated Dgk expressed in E. coli RZ6 drastically reduced its enzymic activity. In their study, the deletion of the $3^{\prime}$ end of the $d g k$ gene resulted in partial fusions with additional five amino acids at the $\mathrm{C}$ terminus of the truncated Dgk protein, whereas in this study the deletion of the $d g k$ gene was immediately followed by a termination codon in E. coli RZ6. The difference in the deletion construct may be the reason for the discrepancy between the results of Lis \& Kuramitsu (2003) and the present study. In addition, we found that as the Dgk homologues were truncated by one amino acid at a time from the $\mathrm{C}$ terminus, the resultant $S$. mutans mutants showed a step-by-step decrease in acid tolerance, corresponding to the decreased kinase activity of the truncated Dgk. These results suggest that the deletion of the $\mathrm{C}$ terminus of the Dgk homologue might directly cause the loss of kinase activity in the Dgk homologue. The most critical active-site residues (five amino acid residues) appear to reside in the second cytoplasmic domain of Dgk in E. coli (Wen et al., 1996). Although these key components are present in the truncated Dgk protein expressed in RZDGK1, the truncated Dgk had no kinase activity. The C-terminal region of $S$. mutans Dgk may harbour residues required for catalysis and/or the loss of kinase activity may be due to incorrect folding of the protein lacking C-terminal residues.

The role of the $d g k$ gene in the cariogenicity of S. mutans was evaluated by in vivo studies. No distinct difference was detected in the development of fissure caries among the three treatment groups. The water control group exhibited fissure caries formation independent of S. mutans. The animal experiment system used in this study seems not to be suitable to evaluate fissure caries formation caused by $S$. mutans. On the other hand, the $d g k$ deletion mutant exhibited a marked reduction in smooth-surface caries compared to that of the wild-type. Furthermore, the rate of smooth-surface caries induction associated with the $d g k$ deletion mutant was similar to that of the water control. These results suggest that the $d g k$ gene might play a critical role in smooth-surface caries formation. Since smoothsurface caries is not as prevalent as fissure caries in humans (Loesche, 1986), the role of $d g k$ in the virulence of $S$. mutans may not be as significant as suggested from our rat model experiments. This is, however, not to detract from our findings but only serves to indicate the relative importance of the results to the human situation.

The infection level of the $d g k$ mutant strain was lower than that of the wild-type strain. Considering the results of the animal experiment, that the $d g k$ mutation did not effectively affect fissure caries formation, but drastically reduced smooth-surface caries formation, decreased sucrosedependent colonization of the $d g k$ mutant may contribute to a decrease in $S$. mutans infection levels. However, in vitro experiments revealed that the $d g k$ mutation did not affect sucrose-dependent biofilm formation at all, while a slight effect on glucose-dependent (without sucrose) biofilm formation was observed (data not shown), consistent with the result of the previous study (Yoshida \& Kuramitsu, 2002). These results suggest that the Dgk may be not involved in sucrose-dependent colonization of S. mutans in vitro. However, the decrease of glucose-dependent biofilm formation of the dgk mutant may affect sucrose-dependent colonization of $S$. mutans in vivo. On the other hand, based on the fact that the $d g k$ mutant lacks the ability to grow at $\mathrm{pH} 5.5$, decreased survival of the $d g k$ mutant within dental plaque may cause a decrease in S. mutans infection levels. In any case, it is not clear whether the $d g k$ gene defect in $S$. mutans affects survival of the organism in the oral cavity or its colonization activity on smooth surfaces.

It is somewhat confusing that infection both with the wildtype strain and with the $d g k$ mutant strain led to reduced smooth-surface plaque extent relative to the water control. However, the measurement of plaque extent shown in Table 4 does not reflect net mass of dental plaque: it depends only on the apparent extent of plaque. Dextran produced by the glucosyltransferases from $S$. mutans may contribute to the formation of the tight plaque, while the plaque formed in the control experiment may be loosely diffused.

Although several aspects remain unclear concerning the mechanism of how the Dgk homologue is involved in cariogenicity of $S$. mutans, our study clearly shows that kinase activity of the Dgk homologue is crucial for $S$. mutans cariogenicity on smooth tooth surfaces.

\section{ACKNOWLEDGEMENTS}

We thank Thomas Thurnheer for advice and Hilary Holmes, Verena Osterwalder and Martin Gander for excellent technical assistance with the animal experiments. This work was supported in part by Grantsin-Aid for Scientific Research 19592408 (Y.S.) and 20659329 (Y.Y.) from the Ministry of Education, Culture, Sports, Science and Technology of Japan.

\section{REFERENCES}

Chen, P., Novak, J., Qi, F. \& Caufield, P. W. (1998). Diacylglycerol kinase is involved in regulation of expression of the lantibiotic mutacin II of Streptococcus mutans. J Bacteriol 180, 167-170. 
Hanahan, D. (1983). Studies on transformation of Escherichia coli with plasmids. J Mol Biol 166, 557-580.

Hasin, M. \& Kennedy, E. P. (1982). Role of phosphatidylethanolamine in the biosynthesis of pyrophosphoethanolamine residues in the lipopolysaccharide of Escherichia coli. J Biol Chem 257, 12475-12477.

Keyes, P. H. (1958a). Dental caries in the molar teeth of rats. I. Distribution of lesions induced by high-carbohydrate low-fat diets. J Dent Res 37, 1077-1087.

Keyes, P. H. (1958b). Dental caries in the molar teeth of rats. II. A method for diagnosing and scoring several types of lesions simultaneously. J Dent Res 37, 1088-1099.

König, K. G., Marthaler, T. M. \& Mühlemann, H. R. (1958). Methodik der kurzfristig erzeugten Rattenkaries. Dtsch Zahn Mund Kieferheilkd 29, 99-127 in German.

Korithoski, B., Lévesque, C. M. \& Cvitkovitch, D. G. (2007). Involvement of the detoxifying enzyme lactoylglutathione lyase in Streptococcus mutans aciduricity. J Bacteriol 189, 7586-7592.

Lis, M. \& Kuramitsu, H. K. (2003). The stress-responsive $d g k$ gene from Streptococcus mutans encodes a putative undecaprenol kinase activity. Infect Immun 71, 1938-1943.

Livak, K. J. \& Schmittgen, T. D. (2001). Analysis of relative gene expression data using real-time quantitative PCR and the $2^{-\triangle \Delta C T}$ method. Methods 25, 402-408.

Loesche, W. J. (1986). Role of Streptococcus mutans in human dental decay. Microbiol Rev 50, 353-380.

Moolenaar, W. H., Kruijer, W., Tilly, B. C., Verlaan, I., Bierman, A. J. \& de Laat, S. W. (1986). Growth factor-like action of phosphatidic acid. Nature 323, 171-173.

Murayama, T. \& Ui, M. (1987). Phosphatidic acid may stimulate membrane receptors mediating adenylate cyclase inhibition and phospholipid breakdown in 3T3 fibroblasts. J Biol Chem 262, 55225529.

Nishizuka, Y. (1984). The role of protein kinase C in cell surface signal transduction and tumour promotion. Nature 308, 693-698.

Perry, D., Wondrack, L. M. \& Kuramitsu, H. K. (1983). Genetic transformation of putative cariogenic properties in Streptococcus mutans. Infect Immun 41, 722-727.

Preiss, J., Loomis, C. R., Bishop, W. R., Stein, R., Niedel, J. E. \& Bell, R. M. (1986). Quantitative measurement of $s n$-1,2-diacylglycerols present in platelets, hepatocytes, and ras- and sis-transformed normal rat kidney cells. J Biol Chem 261, 8597-8600.

Quivey, R. G., Kuhnert, W. L. \& Hahn, K. (2001). Genetics of acid adaptation in oral streptococci. Crit Rev Oral Biol Med 12, 301-314.

Raetz, C. R. \& Newman, K. F. (1978). Neutral lipid accumulation in the membranes of Escherichia coli mutants lacking diglyceride kinase. J Biol Chem 253, 3882-3887.

Raetz, C. R. \& Newman, K. F. (1979). Diglyceride kinase mutants of Escherichia coli: inner membrane association of 1,2-diglyceride and its relation to synthesis of membrane-derived oligosaccharides. J Bacteriol 137, 860-868.
Regolati, B. \& Hotz, P. (1972). Cariostatic effect of glycerophosphate. Helv Odontol Acta 16, 13-18.

Rotering, H. \& Raetz, C. R. (1983). Appearance of monoglyceride and triglyceride in the cell envelope of Escherichia coli mutants defective in diglyceride kinase. J Biol Chem 258, 8068-8073.

Sambrook, J. \& Russell, D. W. (2001). Molecular Cloning: a Laboratory Manual, 3rd edn. Cold Spring Harbor, NY: Cold Spring Harbor Laboratory.

Sanders, C. R., II, Czerski, L., Vinogradova, O., Badola, P., Song, D. \& Smith, S. O. (1996). Escherichia coli diacylglycerol kinase is an $\alpha$ helical polytopic membrane protein and can spontaneously insert into preformed lipid vesicles. Biochemistry 35, 8610-8618.

Senadheera, M. D., Guggenheim, B., Spatafora, G. A., Huang, Y. C., Choi, J., Hung, D. C., Treglown, J. S., Goodman, S. D., Ellen, R. P. \& Cvitkovitch, D. G. (2005). A VicRK signal transduction system in Streptococcus mutans affects $g t f B C D, g b p B$, and $f t f$ expression, biofilm formation, and genetic competence development. J Bacteriol 187, 4064-4076.

Shibata, Y., Yamashita, Y., Nakano, Y. \& Koga, T. (1999). Isolation and characterization of the $\mathrm{rml}$ gene homologs from Porphyromonas gingivalis. Oral Microbiol Immunol 14, 339-347.

Shibata, Y., Yamashita, Y., Ozaki, K., Nakano, Y. \& Koga, T. (2002). Expression and characterization of streptococcal rgp genes required for rhamnan synthesis in Escherichia coli. Infect Immun 70, 28912898.

Shiroza, T. \& Kuramitsu, H. K. (1993). Construction of a model secretion system for oral streptococci. Infect Immun 61, 3745-3755.

Smith, R. L., O'Toole, J. F., Maguire, M. E. \& Sanders, C. R., II (1994). Membrane topology of Escherichia coli diacylglycerol kinase. J Bacteriol 176, 5459-5465.

Tanzer, J. M., Livingston, J. \& Thompson, A. M. (2001). The microbiology of primary dental caries in humans. J Dent Educ 65, 1028-1037.

Topham, M. K. \& Prescott, S. M. (1999). Mammalian diacylglycerol kinases, a family of lipid kinases with signaling functions. J Biol Chem 274, 11447-11450.

Walsh, J. P., Loomis, C. R. \& Bell, R. M. (1986). Regulation of diacylglycerol kinase biosynthesis in Escherichia coli. A trans-acting $d g k R$ mutation increases transcription of the structural gene. J Biol Chem 261, 11021-11027.

Wen, J., Chen, X. \& Bowie, J. U. (1996). Exploring the allowed sequence space of a membrane protein. Nat Struct Biol 3, 141-148.

Yamashita, Y., Takehara, T. \& Kuramitsu, H. K. (1993). Molecular characterization of a Streptococcus mutans mutant altered in environmental stress responses. J Bacteriol 175, 6220-6228.

Yoshida, A. \& Kuramitsu, H. K. (2002). Multiple Streptococcus mutans genes are involved in biofilm formation. Appl Environ Microbiol 68, 6283-6291.

Edited by: M. Kilian 